\title{
Plagas asociadas a los cítricos en Chinandega, Nicaragua, 2018
}

\author{
Pests associated with citrus in Chinandega, Nicaragua, 2018
}

Edgardo Jiménez-Martínez ${ }^{1}$

Elber Méndez López ${ }^{2}$

\section{Resumen}

Los cítricos (Citrus spp. L.) representan en Nicaragua una fuente de divisa y generación de empleo porque, no existe un reporte formal sobre la ocurrencia poblacional de insectos asociados a los cítricos. El presente estudio tuvo el propósito de determinar la fluctuación poblacional de insectos plagas y benéficos en cítricos. El estudio se realizó en los municipios de Chinandega y Chichigalpa, departamento de Chinandega, en el período de abril a septiembre del 2014, el levantamiento de datos fue en seis fincas, en cada finca se ubicaron seis trampas, tres trampas de caída libre (Pitfall traps) para la captura de insectos rastreros, y tres trampas de galón con melaza para la captura de insectos voladores, los insectos colectados se enviaron al laboratorio de entomología de la UNA para su identificación hasta el nivel de especie, se evaluó el número de insectos por familia y a los datos se le realizó un análisis de varianza, la diversidad y abundancia se estimó utilizando el índice de diversidad Shannon-Weaver, para las diferentes familias de la población. Los resultados obtenidos demostraron que las principales familias de insectos asociados al cultivo de cítricos son, Formicidae, Syrphidae, Vespidae, Tachinidae, Carabidae, Apidae, Tenebrionidae, Chrysopidae, Sarcophagidae y de los arácnidos se encontró a la familia Araneidae, se concluye que La mayor riqueza de insectos asociados a los cítricos fue encontrada en la finca Farid seguida de las fincas Mariaelsa y Pañueleta, esto es debido a baja aplicación de pesticida, presencia de abundante vegetación silvestre y cercanía con otras especies de cultivadas en la zona .

Palabras clave: fluctuación poblacional; artrópodos; riqueza; abundancia

\section{Abstract}

Citrus fruits (Citrus spp. L.) represent a source of currency and employment generation in Nicaragua, because there is no formal report on the population occurrence of insects associated with citrus fruits. The present study had the purpose of determining the population fluctuation of pests and beneficial insects in citrus fruits. The study was carried out in the municipalities of Chinandega and Chichigalpa, Chinandega

\footnotetext{
PhD en Entomología, Docente Investigador, Director de Investigación, Extensión y Posgrado, Universidad Nacional Agraria, Nicaragua. Correo: edgardo.jimenez@ci.una.edu.ni ORCID: https://orcid.org/oooo-ooo3-1086-7380

2 MSc en Sanidad Vegetal. Instituto de Protección y Sanidad Agropecuaria (IPSA). Correo, elbermendez@yahoo.com
}

Recibido: 08/01/2021 - Aprobado: 05/02/2021

JJiménez Martínez, E., \& Méndez López, E. (2021). Plagas asociadas a los cítricos en Chinandega, Nicaragua, 2018. Ciencia E Interculturalidad, 28(01), 138 - 150. https://doi.org/10.5377/rci.v28i01.11465 
Department, from April to September 2014, the data collection was in six farms, in each farm six traps were located, three free fall traps (Pitfall traps) for the capture of crawling insects, and three gallon traps with molasses for the capture of flying insects, the collected insects were sent to the Entomology Laboratory at UNA for their identification down to the species level, the number of insects was evaluated by family and the data was performed in a variance analysis, the diversity and abundance were estimated using the Shannon-Weaver diversity index, for the different families of the population. The results obtained showed that the main families of insects associated with citrus cultivation are Formicidae, Syrphidae, Vespidae, Tachinidae, Carabidae, Apidae, Tenebrionidae, Chrysopidae, Sarcophagidae and from arachnids, the Araneidae family was found, it is concluded that the largest wealth of insects associated with citrus fruits was found in the Farid farm followed by the Mariaelsa and Pañueleta farms, this is due to low pesticide application, presence of abundant wild vegetation and proximity to other species cultivated in the area.

Keywords: population fluctuation; arthropods; wealth; abundance.

\section{Introducción}

Los cítricos constituyen un género (Citrus) que, comprenden parte de la familia de las rutáceas (Rutaceae), conformado por varias especies, entre ellas, naranjas (Citrus sinensis, Citrus aurantium), limones (Citrus limón), mandarinas (Citrus reticulata, Citrus reshni) y pomelos (Citrus paradisi Macf). El origen del género Citrus se sitúa en el sureste de Asia y el centro de China, Filipinas y el archipiélago Indomalayo hasta Nueva Guinea. Los cítricos son cultivos permanentes y en general tienen capacidad de adaptación a climas muy diversos, pero la textura ideal de los suelos para su cultivo está comprendida entre liviana y media (Roldan y Salazar, 2002).

La problemática fitosanitaria de los cítricos está relacionada con insectos vectores de microorganismos como fitoplasmas, bacterias y virus. Según el informe anual del Instituto de Protección y Sanidad Agropecuaria (IPSA, 2015), Diaphorina citri (Psilidae) y Brevipalpus spp., (Acaridae) son las principales plagas en los cítricos de Nicaragua y vectores del dragón amarillo (Huanglongbing) y Leprosis (Fitoplasma) de los cítricos, ocasionándole daños e incidiendo en el rendimiento productivo del cultivo.

Los cultivos de cítricos albergan diversas especies vegetales y animales, entre ellas insectos dañinos y benéficos que, generalmente, se encuentran en equilibrio ecológico y en poblaciones estables, lo que facilita el establecimiento, reproducción y acción de depredadores, parasitoides y patógenos de los insectos dañinos (Jiménez-Martínez y Rodriguez, 2014).

Con esta investigación se pretende obtener una base de conocimiento científico sobre la identificación de los principales insectos asociados al cultivo de cítricos en 
Nicaragua. Información que podrá ser utilizada por los productores e investigadores nacionales que deseen hacer uso de esta herramienta, ya que en nuestro país muy poco se conoce sobre la diversidad de insectos que intervienen o interactúan en el ambiente del cultivo.

En Nicaragua, se conocen y se han reportado algunos insectos plagas que afectan a los cítricos, pero no se ha publicado un documento científico donde se describa la dinámica poblacional de insectos asociados que influyan directamente en este cultivo. El propósito de este estudio es generar conocimiento científico y ser una herramienta en la identificación de las principales familias, abundancia, diversidad, fluctuación poblacional y descripción del comportamiento de las especies insectiles asociadas a los cítricos. Información que podrá ser utilizada por los productores nacionales en el diseño de un mejor plan de manejo agronómico y fitosanitario en este cultivo.

\section{Materiales y métodos}

\section{Ubicación del estudio}

El estudio se desarrolló de marzo a septiembre del 2014 en seis fincas de cítricos, ubicadas en los municipios de Chichigalpa y Chinandega, departamento de Chinandega, tres de las fincas están ubicadas en el municipio de Chichigalpa, finca María Elsa, productor Silvio Velásquez Amaya y finca Los Panchos, productor Francisco Ortega en la comunidad de Cosmapa y en la comunidad El Pellizco finca Farid productor Farid Eleazar.

En el municipio de Chinandega se seleccionaron las fincas en la comunidad de La Grecia $\mathrm{N}^{\circ}$ 4, finca Deysi de la productora Nubia Baca Martínez y en la comunidad La Concepción, finca Pañueleta, productor Cesar Castillo y finca El Cruceiro del productor Salvador Romero.

\section{Diseño metodológico para el muestreo en campo}

En cada finca se ubicaron dos tipos de trampa y tres sitios de muestreo, el muestreo se realizó cada quince días. Para el estudio de artrópodos que se desplazan por el suelo se utilizaron trampas de caída libre o Pitfall traps, debido a su efectividad y simplicidad (Tadashi, 2004). Trampas de galones plásticos con agua y melaza para la captura de insectos voladores, método utilizado por Löhr y Parra (2014) para captura masiva del picudo negro de las palmas (Rhynchophorus palmarum), por Rugama y López (2011) para captura de insectos rastreros asociados al marañón (Anacardium occidentale L.) y por Porres (2017) para el control de mosca tipo Fungus Gnat en plantaciones de helechos ornamentales. 


\section{Procesamiento de muestras e identificación de insectos en laboratorio}

En el laboratorio los insectos fueron contados y registrados en base dato en hoja Excel. Identificados y separados por orden, familia, género y algunos hasta especie, consultando la literatura de Borror, De Long y Triplehorn (1979), Hernández-Ortiz (1992) y King y Sanders (1989).

Variables evaluadas

- Abundancia total de insectos encontrados por finca.

- Abundancia total de insectos y arañas encontrados por familia.

- Riqueza de insectos encontrados por géneros.

- Cálculo de índice de diversidad de insectos.

- Fluctuación poblacional de insectos de la familia Formicidae, Syrphidae, Vespidae, Tachinidae.

- Fluctuación poblacional de arácnidos.

\section{Cálculo del índice de diversidad de insectos}

La diversidad de insectos fue determinada de acuerdo con la metodología utilizada por Vásquez et al. (2009), basados en el índice de diversidad Shannon-Weaver.

\section{Análisis estadístico de los datos}

Los datos de insectos fueron analizados de acuerdo con la metodología utilizada por Barrios et al. (2004), Urías López et al. (2007), Rúgama y López (2011), Lacayo y Mayorga (2014). Después de colectados los insectos fueron agrupados por variables de familias de insectos por finca en una base de datos en EXCEL, cada variable fue comparada entre fincas, efectuando un análisis de varianza utilizando el programa SAS (2003). El nivel de significancia usado fue de $(\mathrm{P}=0.05)$. 


\section{Resultados y discusión}

Cuadro no. 1: Diversidad de insectos y arañas identificados en cítricos

\begin{tabular}{|c|c|c|c|c|c|}
\hline Orden & Familia & Género & Especie & Nombre Común & Hábito Alimenticio \\
\hline \multirow{8}{*}{ Hymenoptera } & \multirow{3}{*}{ Formicidae } & Atta & $s p$ & zompopo & Defoliador \\
\hline & & Camponotus & $s p$ & Hormiga & Depredador \\
\hline & & Atta & cephalotes & Zompopo & Defoliador \\
\hline & \multirow{2}{*}{ Apidae } & Apis & mellifera & Abeja & Polinizador \\
\hline & & Euplusia & $s p$ & Abeja & Polinizador \\
\hline & Halictidae & Halictus & $s p$ & Piojo de las hortalizas & Chupador \\
\hline & \multirow{2}{*}{ Vespidae } & Polybia & $s p$ & Avispa carnera & Depredador de larvas pequeñas \\
\hline & & Polistes & $s p$ & Cátala & Depredador \\
\hline \multirow{7}{*}{ Diptera } & Tachinidae & Lespesia & $s p$ & Tachinidos & Parasitoides de larvas \\
\hline & Calliphoridae & Cochliomyia & $s p$ & Gusano de las heridas & $\begin{array}{l}\text { Se alimenta de carroña, excre- } \\
\text { mento y sangre }\end{array}$ \\
\hline & Muscidae & Musca & domestica & Mosca & $\begin{array}{l}\text { Se alimenta de materia descom- } \\
\text { puesta }\end{array}$ \\
\hline & Syrphidae & Allograpta & $s p$ & Mosca de las flores & Polinizador \\
\hline & Otitidae & Euxesta & $s p$ & Mosca Zebra & Ataca grano y tallo de maíz \\
\hline & Sarcophagidae & Sarcophaga & $s p$ & Mosca & Parasitoide \\
\hline & Dolichopodidae & Condylostrylus & $s p$ & & $\begin{array}{c}\text { Se alimenta de presa cuerpo } \\
\text { suave }\end{array}$ \\
\hline \multirow{10}{*}{ Coleóptera } & \multirow{2}{*}{ Elateridae } & Aeolus & $s p$ & Gusano alambre & Ataca raíces \\
\hline & & Conoderus & $s p$ & Gusano alambre & Ataca raíces \\
\hline & \multirow{4}{*}{ Scarabaeidae } & Phyllophaga & $s p$ & Escarabajo & Las larvas se alimentan de raíces \\
\hline & & Euphoria & $s p$ & Escarabajo & Las larvas se alimentan de raíces \\
\hline & & Lygirus & $s p$ & Escarabajo & Degradan materia orgánica \\
\hline & & Copris & $s p$ & Escarabajo & Degradan materia orgánica \\
\hline & \multirow[b]{2}{*}{ Tenebrionidae } & Selenophorus & $s p$ & $\begin{array}{c}\text { Escarabajo Gusano } \\
\text { de alambre }\end{array}$ & Plaga del suelo Ataca raíces \\
\hline & & Epitragus & $s p$ & $\begin{array}{l}\text { Escarabajo Falso } \\
\text { gusano de alambre }\end{array}$ & Plaga del suelo Ataca raíces \\
\hline & Cucurlionidae & Pantomorus & femoratus & Picudo & Defoliador \\
\hline & Carabidae & Calosoma & $s p$ & Escarabajo & Depredador \\
\hline \multirow{3}{*}{ Coleóptera } & Buprestidae & Chrysobotris & $s p$ & Escarabajo & Barrenador de madera \\
\hline & Campiridae & Aspisoma & $s p$ & Luciérnaga & Depredador \\
\hline & Cerambicidae & Stenocorus & $s p p$ & Escarabajo & Barrenador \\
\hline
\end{tabular}




\begin{tabular}{|c|c|c|c|c|c|}
\hline Orden & Familia & Género & Especie & Nombre Común & Hábito Alimenticio \\
\hline \multirow{2}{*}{ Homóptera } & \multirow{2}{*}{ Cicadellidae } & Draeculacephala & $s p$ & Salta hoja & chupador \\
\cline { 3 - 6 } & Oncometopia & $s p$ & Salta hoja & chupador \\
\hline Blattodea & Blattellidae & Blattella & $s p$ & cucaracha & Vector de enfermedades \\
\hline \multirow{4}{*}{ Lepidóptera } & Hesperidae & Urbanus & proteus & Gusano cabezón & Defoliador \\
\cline { 2 - 6 } & Noctuidae & Spodoptera & frugiperda & Gusano cogollero & Defoliador \\
\cline { 2 - 6 } & Pyralidae & Diatraea & $s p p$ & Papalote & barrenador \\
\hline Hemiptera & Cydnidae & Pangaeus & $s p$ & Maya hedionda & Se alimenta de raíces \\
\hline Aracnida & Aranidae & Cynorta & $s p$ & Araña & Depredadores \\
\hline
\end{tabular}

En este estudio se encontraron nueve órdenes, 26 familias y 36 especies. Se conoce que los defoliadores, succionadores de floema y barrenadores son considerados plagas por los daños que realizan en las plantas al alimentarse, en este estudio las especies con estos hábitos representan $47.22 \%$, mientras que las especies consideradas benéficas con hábitos depredadores, parasitoides, polinizadores y degradadores de materia orgánica representan el $52.78 \%$, considerados los de mayor importancia en la entomofauna asociada a cítricos (Cuadro 1).

\section{Abundancia total de insectos encontrados por finca}

La abundancia total fue de 3523 . Siendo la finca María Elsa la que presentó mayor número de insectos (Figura 1). La abundancia de insectos encontrados en la finca María Elsa, se debe al tipo de manejo agronómico, el cual contempla baja aplicación de pesticidas, poca actividad de mantenimiento y la presencia de otros cultivos como cucurbitáceas, tomate y maíz. En los alrededores de estas fincas existían cultivos como caña de azúcar y maní, lo que contribuye a la mayor presencia de insectos por familia, por la diversidad de cultivos, que representa mayor cantidad y diversidad de insectos.

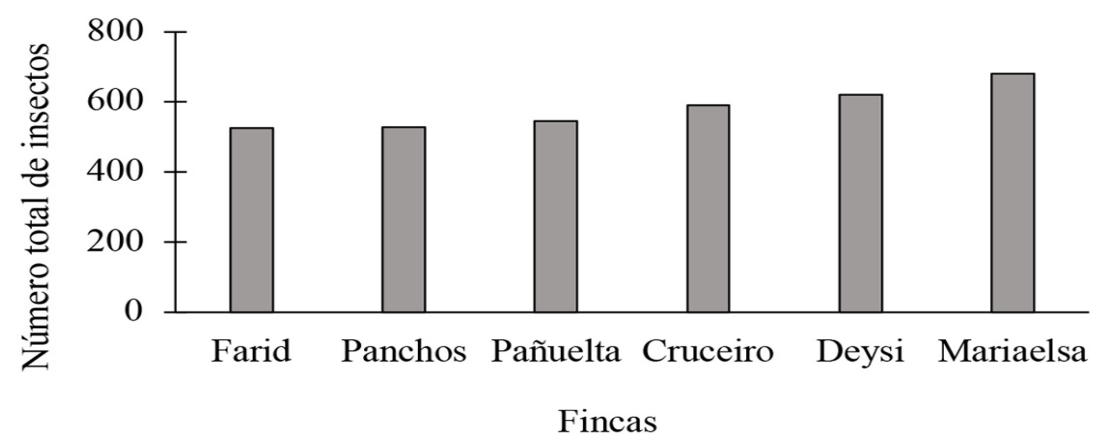

Figura no. 1. Abundancia total de insectos encontrados por finca de cítricos. 


\section{Abundancia total de insectos y arañas por familia}

La familia Formicidae presento mayor cantidad de especímenes, seguido de Syrphidae y Vespidae, otra familia como los Tachinidae y Araneidae (clase arácnida, artrópodos) presentaron 112 especímenes cada uno, el orden de ocurrencia de cada familia y la abundancia registrada para cada una se muestra en la Figura 2.

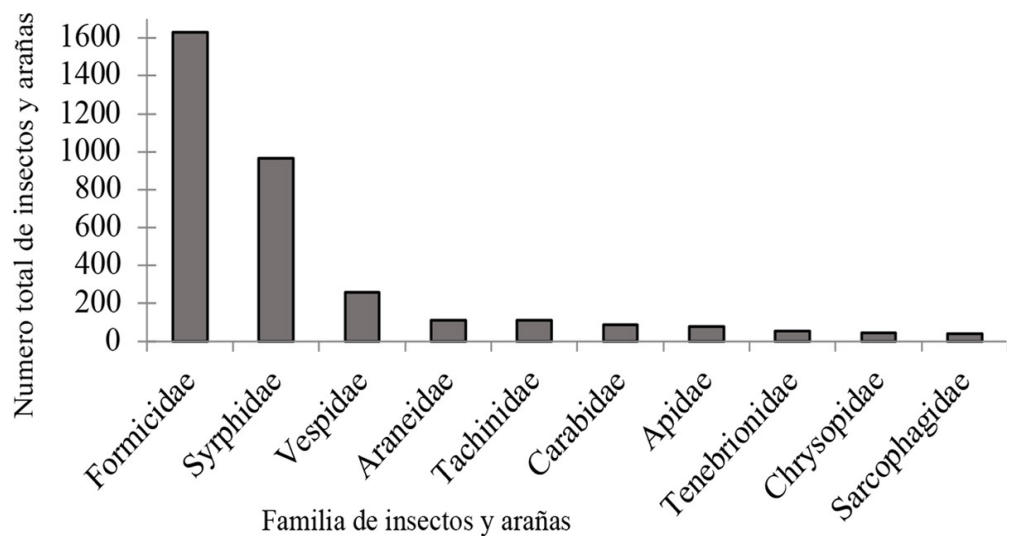

Figura no. 2. Abundancia de insectos y arañas por familia encontrados en el cultivo de cítricos.

\section{Riqueza de insectos encontrados por género}

Moreno (2001) menciona que, la riqueza de insectos es el número de especies presentes, contando con un inventario completo que nos permita conocer el número total de especies obtenidas por un censo de la comunidad. La riqueza total de géneros se presenta en la Figura 3, la finca Farid presento la mayor riqueza de géneros seguido de la finca Mariaelsa, Pañueleta, Los panchos y El Cruceiro

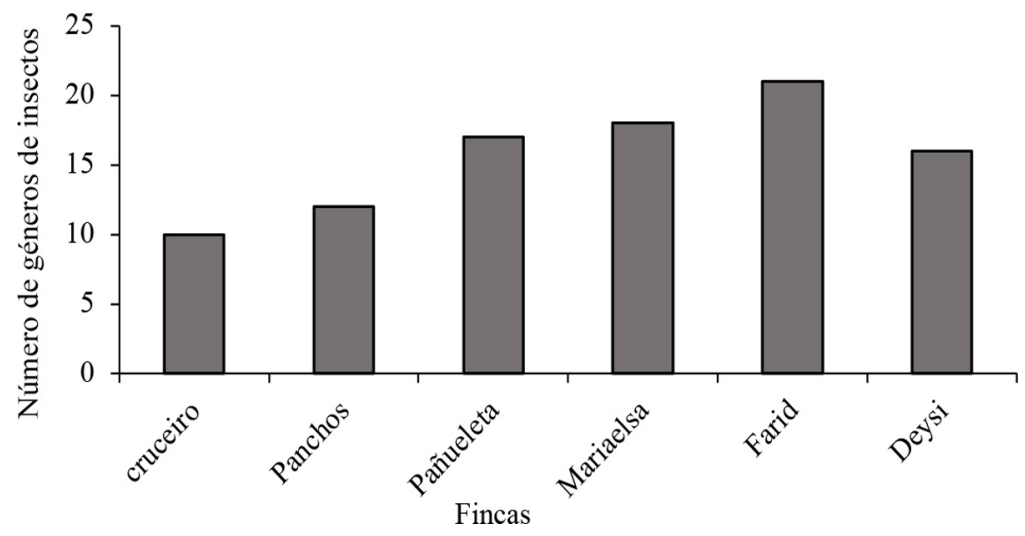

Figura no. 3. Riqueza de géneros de insectos y arañas encontrados en seis fincas con cultivo de cítrico. 
La mayor presencia de géneros en las fincas Farid, Mariaelsa, Pañueleta y Deysi se debe a la cercanía de cultivos como maíz, frijoles, cucurbitáceas, cañas, maní y yuca. Estos cultivos contribuyen a la mayor presencia de géneros y la emigración hacia el cultivo de cítricos por lo que son capturados en las trampas por caída natural o por la fuerza del viento.

\section{Índice de diversidad de Shannon-Weaver}

La familia con mayor índice de diversidad fue Formicidae seguido de Syrphidae, ambas registradas en las fincas Farid, Mariaelsa, Panchos y Deysi. El índice de biodiversidad más bajo fue de la familia Carabidae en la finca Deysi (Cuadro 4). Los índices de diversidad obtenidos en las fincas (1.00 a 1.44) son inferiores a los establecidos por Shannon-Weaver, 1948, cuyos valores normales están entre 2 y 3, valores inferiores a 2 se consideran bajos y superiores a 3 son altos.

Cuadro no. 4: Índice de diversidad Shannon-Weaver de las principales familias de insectos asociados al cultivo de cítricos

\begin{tabular}{|l|l|l|l|l|l|l|}
\hline \multirow{2}{*}{ Familias de insectos } & \multicolumn{7}{|c|}{ Índice de diversidad de Shannon-Weaver por finca } \\
\cline { 2 - 7 } & \multicolumn{1}{|c|}{ Farid } & \multicolumn{1}{|c|}{ Mariaelsa } & \multicolumn{1}{c|}{ Panchos } & \multicolumn{1}{c|}{ Deysi } & \multicolumn{1}{c|}{ Pañueleta } & \multicolumn{1}{c|}{ Cruceiro } \\
\hline Formicidae & 1,44 & 1,44 & 1,41 & 1,44 & 1,41 & 1,42 \\
\hline Syrphidae & 1,43 & 1,44 & 1,37 & 1,44 & 1,35 & 1,41 \\
\hline Vespidae & 1,19 & 1,19 & 1,24 & 1,22 & 1,21 & 1,21 \\
\hline Aracnidae & 1,09 & 1,15 & 1,11 & 1,12 & 1,08 & 1,13 \\
\hline Tachinidae & 1,12 & 1,11 & 1,16 & 1,09 & 1,11 & 1,11 \\
\hline Carabidae & 1,09 & 1,11 & 1,04 & 1,00 & 1,23 & 1,05 \\
\hline Apidae & 1,09 & 1,05 & 1,10 & 1,09 & 1,05 & 1,15 \\
\hline Tenebrionidae & 1,16 & 1,06 & 1,04 & 1,04 & 1,03 & 1,05 \\
\hline Chrysopidae & 1,08 & 1,04 & 1,06 & 1,05 & 1,03 & 1,09 \\
\hline Sarcophagidae & 1,06 & 1,05 & 1,08 & 1,03 & 1,04 & 1,05 \\
\hline
\end{tabular}

\section{Fluctuación poblacional de insectos}

El mayor número de estos insectos lo presentaron las fincas Cruceiro, Pañueleta, Los Panchos y Mariaelsa, el menor número de estos insectos se registró en las fincas Deysi y Farid. 
Cuadro no. 5: Análisis de varianzas del número de insectos de las familias Formicidae, Syrphidae, Vespidae y Tachinidae en el cultivo de cítricos

\begin{tabular}{|l|l|l|l|l|}
\hline \multicolumn{1}{|c|}{ Fincas } & \multicolumn{1}{|c|}{$\begin{array}{c}\text { Número insectos de } \\
\text { familia Formicidae }\end{array}$} & \multicolumn{1}{|c|}{$\begin{array}{c}\text { Múmero insectos de familia } \pm \text { ES } \\
\text { Syrphidae }\end{array}$} & \multicolumn{1}{c|}{$\begin{array}{c}\text { Múmero insectos de familia } \pm \text { ES } \\
\text { Vespidae }\end{array}$} & \multicolumn{1}{c|}{$\begin{array}{c}\text { Número insectos de familia } \\
\text { Tachinidae }\end{array}$} \\
\hline Cruceiro & $4.96 \pm 1.49 \mathrm{a}$ & $4.02 \pm 0.60 \mathrm{ab}$ & $1.19 \pm 0.09 \mathrm{~b}$ & \multicolumn{1}{c|}{ Media $\pm \mathrm{ES}$} \\
\hline Pañueleta & $4.57 \pm 0.94 \mathrm{a}$ & $2.52 \pm 0.35 \mathrm{C}$ & $1.48 \pm 0.25 \mathrm{ab}$ & $1.54 \pm 0.20 \mathrm{a}$ \\
\hline Panchos & $4.38 \pm 0.69 \mathrm{a}$ & $2.44 \pm 0.30 \mathrm{C}$ & $1.72 \pm 0.24 \mathrm{a}$ & $1.73 \pm 0.22 \mathrm{a}$ \\
\hline Mariaelsa & $4.26 \pm 0.59 \mathrm{a}$ & $5.21 \pm 1.79 \mathrm{a}$ & $1.24 \pm 0.08 \mathrm{ab}$ & $1.70 \pm 0.21 \mathrm{a}$ \\
\hline Deysi & $3.98 \pm 1.04 \mathrm{~b}$ & $4.42 \pm 0.60 \mathrm{ab}$ & $1.35 \pm 0.11 \mathrm{ab}$ & $1.40 \pm 0.22 \mathrm{a}$ \\
\hline Farid & $3.23 \pm 0.35 \mathrm{~b}$ & $3.41 \pm 0.81 \mathrm{~b}$ & $1.35 \pm 0.10 \mathrm{ab}$ & $1.33 \pm 0.14 \mathrm{a}$ \\
\hline $\mathrm{ds} ; \mathrm{C.V}$ & $7.09 ; 167.28$ & $6.26 ; 166.35$ & $0.86 ; 62.6637$ & $0.66 ; 44.74$ \\
\hline P & $0.0463 \mathrm{DS}$ & $0.0281 \mathrm{DS}$ & $0.02882 \mathrm{DS}$ & $0.2886 \mathrm{NS}$ \\
\hline F; df; $\mathrm{n}$ & $0.38 ; 358 ; 364$ & $1.26 ; 239 ; 245$ & $1.25 ; 160 ; 166$ & 1.27 \\
\hline
\end{tabular}

ES= Error estándar, DS = Diferencia Significativa, C.V = Coeficiente de Variación, $\mathrm{P}=$ Probabilidad según Duncan, $\mathrm{F}=$ Fisher calculado df = Grados de libertad del error, $\mathrm{n}=$ Números de datos utilizados en el aná-

lisis. ${ }^{*}$ Medias con letras distintas existe diferencias significativas

\section{Fluctuación poblacional de insectos de la familia Formicidae}

Los insectos de la familia Formicidae juegan un importante papel en los ecosistemas como transportadoras de semillas, depredadoras de artrópodos y mejoradoras de la estructura del suelo. Esta familia se encontró en casi todas las partes del árbol, fueron los insectos con mayor presencia en las fechas de muestreo, pero no se observaron causando daños, por lo que no se consideran especies plagas en cítricos. Las hormigas se encuentran entre los artrópodos más abundantes en el agrosistema de los cítricos, su acción puede influir en las poblaciones de insectos fitófagos al interferir en su control biológico (Martínez Ferrer et al., 2010) desarrollan una actividad depredadora para varias especies de insectos, entre las que podemos citar Hesperidae, Noctuidae, Pyralidae, Buprestidae, Carabidae, Cucurlionidae, Scarabaeidae, entre otras.

Las especies más comunes en cítricos son Atta sp., Atta cephalotes y Camponotus $s p$. Estos insectos tienen un tamaño pequeño a grande (1-30mm), cuerpo delgado, antenas con 6-13 segmentos (Sáenz y De la Llana, 1990) son insectos sociales que viven en colonias, tienen una o más reinas y muchas obreras (Jiménez Martínez, 2009). Los representantes son conocidos como hormigas cortadoras o zompopos, cortan pedazos de hojas que llevan al nido para cultivar el hongo del cual se alimentan, algunas especies de esta familia protegen a insectos plagas productoras de mielecilla como mosca blanca y pulgones (Nunes y Dávila, 2004). 


\section{Fluctuación poblacional de insectos de la familia Syrphidae}

El mes con menos incidencia de insectos de la familia Syrphidae fue abril y la finca con menor incidencia La Pañueleta, la presencia de insectos de esta familia fue mayor en la finca Mariaelsa.

Los insectos de esta familia tienen tamaño pequeño a grande de 2 a $25 \mathrm{~mm}$, generalmente y, se asemejan a abejas o avispas, poseen cabeza bastante grande (Sáenz y De la Llana, 1990). Comúnmente llamados moscas de las flores, son de gran importancia económica en la citricultura, los adultos son polinizadores de gran relevancia y pueden desempeñar el papel de las abejas, las larvas de la subfamilia Syrphinae son depredadores de muchas plagas como áfidos (Hemiptera:Aphididae), cóccidos, trips y larvas de mariposas; sin embargo, algunos sírfidos son perjudiciales, las larvas de algunas especies de los géneros Merodon y Eumerus atacan bulbos y tubérculos de plantas ornamentales y legumbres (Barranco, 2003).

\section{Fluctuación poblacional de insectos de la familia Vespidae}

En la comparación del promedio de insectos por planta de la familia Vespidae se encontró que, en la finca Los Panchos se registró la mayor cantidad de insectos y la menor en la finca El Cruceiro.

Esta familia presenta tamaño de mediano a grande $(10-30 \mathrm{~mm})$ con expansión alar entre 18 a $55 \mathrm{~mm}$, cuerpo moderadamente robusto, color generalmente negro y amarillo, alas delanteras con tres celdas su marginales, dobladas en posición de descanso. Son depredadores de plagas de cultivos e importantes agentes polinizadores (Sáenz y De la Llana, 1990). No se considera plaga para el cultivo de cítrico, en los muestreos se observaron en ramas y polinizando las flores. (Davies no aparece en la lista de referencias).

Los véspidos vivientes (Hymenoptera:Vespidae) están conformados por las subfamilias Euparagiinae, Masarinae, Eumeninae, Stenogastrinae, Vespinae y Polistinae. Las tres primeras poseen un modo de vida solitario o primitivamente social, mientras que las tres últimas presentan un comportamiento social más elaborado y comparten características como la construcción de nidos complejos, donde viven varias generaciones de adultos y realizan actividades centrales de la colonia como la reutilización de las celdas de cría, cuidado de los inmaduros hasta emerger en adultos, aprovisionamiento de presas masticadas para sus crías, trofalaxis entre adulto-adulto o adulto-larva y división temporal reproductiva del trabajo (López et al., 2013). 


\section{Fluctuación poblacional de insectos de la familia Tachinidae}

Al realizar el análisis de varianza no se encontró diferencia significativa en la cantidad de insectos encontrados, el mayor número de estos insectos lo presentaron las fincas Los Panchos y Mariaelsa y la finca con menor cantidad fue el Cruceiro.

Estos insectos son de tamaño pequeño a grande (o.5-65mm), cuerpo con forma variable, boca chupadora lamedora con antenas con tres o 39 segmentos, filiformes, moniliformes, plumosas, ojos compuestos generalmente grandes, ocelos generalmente presentes y un par de alas (Sáenz y De la Llana, 1990). Las moscas Tachinidae son importantes polinizadores en gran variedad de plantas.

\section{Conclusiones}

Las principales familias de insectos y arañas encontrados asociados a los cítricos fueron Formicidae, Syrphidae, Vespidae, Tachinidae, y Araneidae.

La mayor abundancia de insectos asociados a los cítricos fue encontrada en las fincas Mariaelsa y Deysi, con mayor presencia de Formicidae y Syrphidae.

La mayor riqueza de insectos asociados a los cítricos fue encontrada en la finca Farid.

\section{Lista de referencia}

Barranco Vega, P. (2003). Dípteros de interés agronómico. Agromícidos, plaga de cultivos hortícolas intensivos. Entomología aplicada, 33(1), 293-307.

Barrios Diaz, B., Alatorre Rosas, R., Calyecac Cortero, H. G., Bautista-Martinez, N. (2004). Identificación y fluctuación poblacional de las plagas de la col (Brassica oleracea var. Capitata) y sus enemigos naturales en Acatzingo, Puebla, MX. Agrociencia, 38:339-248.

Borror, D., De Long, D., Triplehorn, C. (1979). An introduction to the study of insects. 5 ed. Philadelhia, US. 928 p.

Hernández-Ortiz, V. (1992). El Género Anastrepha spp. (Schiner 1868) en México (Diptera: Tephritidae). Taxonomía, distribución y sus plantas huéspedes. Instituto de Ecología, Sociedad Mexicana de Entomología. MX. 161 p.

Instituto de Protección y Sanidad Agropecuaria (2015). Informe anual 2015 de vigilancia fitosanitaria. IPSA, DISAVE-SEMILLAS. (Dirección de Sanidad Vegetal y Semillas), Managua, NI. 
Jiménez- Martínez E. Rodriguez- Flores O, (2014). Universidad Nacional Agraria. Insectos: Plagas de cultivos en Nicaragua. se. Managua, NI. 218 p.

Jiménez-Martínez, E. (2009). Entomología. Universidad Nacional Agraria (UNA). Dirección de Investigación Extensión y Posgrado (DIEP). Editronic, Managua, NI. 112 p.

King, A., y Sanders, J. (1989). Las plagas invertebradas de cultivos anuales alimenticios en América Central. CATIE. Turrialba. 182 p.

Lacayo, R., y Mayorga, J. (2014). Abundancia, riqueza y diversidad insectil asociada al cultivo de Marango (Moringa oleifera L.). (Tesis de grado). Managua, NI. Universidad Nacional Agraria. $56 \mathrm{p}$.

Löhr, B., y Parra, P. P. (2014). Manual de trampeo del picudo negro de las palmas, Rhynchophorus palmarum, en trampas de feromona adaptadas a la situación particular de pequeños productores de la costa del pacífico colombiano. Cali, Centro Internacional de Agricultura Tropical (CIAT). https://cgspace.cgiar.org/ bitstream/handle/10568/56946/Manual\%2ode\%20Trampeo\%2oPicudo\%2o Negro\%2ode\%2olas\%2oPalmas-WEB.pdf

López, Y., Canchila, S., y Álvarez, D. (2013). Listado de avispas sociales (Vespidae: Polistinae) del departamento de Sucre, Colombia. Instituto de Investigación de Recursos Biológicos Alexander von Humboldt. Biota Colombiana, 14(2), 108-113.

Martínez Ferrer, M., Campos Rivela, J., Fibla Queralt, J., y Audi, J. P. (2010). Las hormigas en los campos de cítricos: su influencia en el control biológico de las plagas y su manejo en el marco de una citricultura sostenible. Instituto Nacional de Investigación y Tecnología Agraria y Alimentaria (INIA). Universidad Politécnica de Valencia, España. 3 p. www.agripa.org/download-doc/61203

Moreno, C. (2001). Métodos para medir la biodiversidad. CYTED, ORCIT/UNESCO y SEA. 1 ed. Zaragoza, España.

Nunes, C., Dávila, M. L. (2004). Taxonomía de las Principales Familias y Subfamilias de Insectos de Interés Agrícola en Nicaragua. UCATSE (Universidad Católica Agropecuaria del Trópico Seco Estelí) Nicaragua.

Porres Bustamante, C. M. (2017). Diseño de trampa para el control de mosca tipo Fungus Gnat en plantaciones de helechos ornamentales. (Tesis de grado). Universidad Rafael Landivar. Facultad de arquitectura y diseño. 
Roldan, D., y Salazar, M. (2002). La cadena de cítricos en Colombia. Ministerio de Agricultura y Desarrollo Rural. Documento de trabajo No. 16. Bogotá, Colombia. $33 \mathrm{p}$.

Rugama Lovo, I. M., López Vílchez, M. E. (2011). Identificación y descripción de los principales insectos rastreros asociados al cultivo del marañón (Anacardium occidentalis L.) orgánico y convencional, en León, Nicaragua. (Tesis de grado). Managua. Universidad Nacional Agraria. 91 p.

Sáenz, M., y De La Llana, A. (1990). Entomología sistemática. Universidad Nacional Agraria. Managua, NI. 225 p.

SAS (2003). SAS Languaje guide for personal computer. University of Nebraska. Cary, NC, USA.V.91

Shannon, C. E., y Weaver, W. (1948). The Mathematical Theory of Communication. Reprinted with corrections from The Bell System Technical Journal Urbana. 27, 379-423, 623-656 http://math.harvard.edu/ ctm/home/text/others/shannon/ entropy/entropy.pdf

Tadashi (mayo-agosto 2004). Las trampas de caída para el estudio de artrópodos terrestres en ecosistemas naturales y agrícolas. Granma Ciencia 20(2). http:// www.grciencia.granma.inf.cu/vol\%2020/2/2016_20_n2_a6.pdf

Urías López, M. A., Salazar-García, S., y Johandsen-Naime, R. (2007). Identificación y fluctuación poblacional de especies de trips (Thysanoptera) en aguacate Hass en Nayarit, México. Revista Chapingo Serie Horticultura, 13 (1), 49-54.

Vásquez, L. L., Matienzo, Y., Simonetti, A., Moreno, D., y Álvarez, A. (2009). Diversidad de especies de hormigas (Hymenoptera: Formicidae) en cafetales afectados por Hypothenemus hampei Ferrari (Coleoptera: Cucurlionidae: Escolytinae). Fitosanidad, 13(3), 163-168. 